\title{
Early Diagnosis of Cardiotoxicity in Patients Undergoing Chemotherapy for Acute Lymphoblastic Leukemia
}

\author{
ANDA MILITARU ${ }^{1}$, SEBASTIAN ZUS ${ }^{2}$, ANCA MARIA CIMPEAN ${ }^{3}$, STELA IURCIUC ${ }^{4}$, \\ PETRU MATUSZ ${ }^{5}$, MIRCEA IURCIUC ${ }^{4}$, DANIEL LIGHEZAN ${ }^{1}$ and MARIUS MILITARU ${ }^{6}$ \\ ${ }^{1}$ Medical Semiology I, Department of Internal Medicine I, ${ }^{2}$ Cardiovascular Disease Institute, \\ ${ }^{3}$ Department of Microscopic Morphology/Histology, Angiogenesis Research Center, \\ ${ }^{4}$ Department of Cardiology, Preventive Medicine and Cardiovascular Rehabilitation, \\ ${ }^{5}$ Department of Anatomy and Embriology, and ${ }^{6}$ Department of Neuroscience - Municipal Emergency Hospital, \\ Victor Babes University of Medicine and Pharmacy, Timisoara, Romania
}

\begin{abstract}
Background/Aim: Chemotherapy-induced cardiotoxicity may be observed during treatment or may cause severe cardiac failure as the main cause of death, even several years after therapy implementation. Herein, the aim was to establish the early diagnosis of cardiotoxicity through the periodic evaluation of the left ventricular $(L V)$ and vascular remodeling parameters, in patients with acute lymphoblastic leukemia (ALL). Materials and Methods: The study population included 35 patients diagnosed with $A L L$, evaluated before and 3 months after starting chemotherapy, measuring systolic and diastolic parameters of the LV and intima-media thickness (IMT), arterial stiffness aortic pulse wave velocity (PWVAo) and ankle-brachial index $(A B I)$. Results: After the first 2 cycles of chemotherapy, all patients experienced a drop in $L V$ ejection fraction (LVEF) $(p<0.001)$, and 12 patients suffered a decrease of $L V E F<50 \%$. The ABI $(p<0.05)$ and the global longitudinal strain $(G L S)(p<0.001)$ decreased, while IMT and PWVAo $(p<0.001)$ increased, proving a subclinical deterioration of the LV function and vascular remodeling. Conclusion: Assessment of cardiovascular risk factors before chemotherapy initiation in ALL patients may be helpful for an early diagnosis of chemotherapy-induced cardiotoxicity, thus contributing to early treatment and a subsequent decrease of death caused by such cardiovascular complications.
\end{abstract}

Correspondence to: Anca Maria Cimpean, MD, Ph.D., Professor of Histology, Department of Microscopic Morphology/Histology, Angiogenesis Research Center, Piata Eftimie Murgu 2, 300041 Timisoara, Romania. Tel: +40 720060955, e-mail: ancacimpean1972@ yahoo.com

Key Words: Cardiotoxicity, vascular rigidity, LV dysfunction, anthracyclines.
The most frequent heart-related adverse effects of chemotherapy are cardiac function alteration and the development of heart failure (1). Of the different classes of chemotherapeutics used in cancer treatment, anthracyclines and their analogues have proven to be the most aggressive towards cardiac function $(2,3)$. Anthracyclines are administered in combination with other drugs for malignant cell destruction in patients diagnosed with acute lymphoblastic leukemia (ALL). The risk of cardiotoxicity depends on the cumulative dose and the interaction with other chemotherapeutics (4). Acute cardiotoxicity can occur even after the administration of the first doses of anthracyclines, and represents a temporary alteration of myocardial contractility, which in most cases is reversible (4). Less than $1 \%$ of patients with different types of cancers treated with anthracyclines develop acute cardiotoxicity (5). The most frequent manifestations of cardiotoxicity appear during treatment or in the first year after, but there have been cases of it appearing 10-20 years after the first dose of chemotherapy $(1,3)$. In patients who develop this late form of cardiotoxicity, the usual presentation is that of a progressive dilated cardiomyopathy. The adverse effects of chemotherapeutics are dose-dependent: the greater the dose, the greater the risk of cardiotoxicity, while combination with radiotherapy additionally increases the risk of a cardiotoxic effect (6). The first sign of myocardial alteration is the decline in left ventricular ejection fraction LVEF. Other echocardiographic parameters, such as longitudinal deformation- global longitudinal strain GLS and vascular remodeling parameters as intima media thickness (IMT), arterial stiffness aortic pulse wave velocity (PWVAo), and ankle-brachial index (ABI) support the early diagnosis of cardiotoxicity (7).

Cardiotoxicity is one of the most severe secondary effects of chemotherapy used in cancer treatment. Most often the alteration of cardiac function manifests itself later in the 
clinical evolution of patients. The more information is known regarding the presence of cardiovascular disease or cardiovascular risk factors in patients undergoing chemotherapy, the more measures can be taken to detect the first manifestations and prevent the development of cardiotoxicity. The appropriate conduct would be to diagnose a cardiac involvement as early as possible, in order to prevent heart failure from occurring in these patients (7). One of the best indicators of cardiotoxicity is a decrease in LVEF $(7,8)$. This parameter can easily be obtained by performing a two-dimensional (2D) echocardiogram. LVEF is not the only echocardiographic parameter that suffers changes, but others too, and their analysis can complete the diagnosis of cardiotoxicity. It is often the case that patients with ALL need thoracic, cervical or cranial radiotherapy, in addition to chemotherapy, so that cardiac investigations need to be associated with periodic vascular investigations (7).

\section{Materials and Methods}

We evaluated 35 patients diagnosed with ALL, admitted to the Haematology Clinic of Timisoara Municipal Emergency Clinical Hospital (Timisoara, Romania), between 2015 and 2018. They were administered polychemotherapy according to the national protocols of treatment in acute leukemia. These therapeutic protocols included prednisone, vincristine, and daunorubicin. Patients included into the study had no previous cardiovascular disease and no cardiovascular risk factors. No patients received cardiological treatment before initial examination, or prior anti-cancer treatment. All 35 patients were evaluated at the start of chemotherapy and then after 3 months (after the administration of two cycles of chemotherapy). The two examinations entailed: history taking, blood pressure (BP) and heart rate (HR) measurement, electrocardiography (ECG), body mass index (BMI) calculation, blood tests, chest X-ray, transthoracic echocardiography and vascular parameter determination (IMT, PWVAo, and ABI). Patients were treated according to the guidelines of the European Society of Hematology. All standards of ethics and good clinical practice have been respected.

Transthoracic echocardiography. For the transthoracic echocardiographic measurements, a General Electric Vivid E9 and a $5 \mathrm{MHz}$ transducer (GE Healthcare, Silverwater, Australia) were used. We applied the method of 2D echocardiography in transverse sections in 2D-guided M-mode, and pulsed wave Doppler analysis of the lateral mitral annulus. The echocardiographic images were obtained in the following views: parasternal long axis, parasternal short axis at the LV level, apical 4, 3 and/or 2 chamber view. Systolic function of the LV was evaluated by measuring LVEF and LV fractional shortening (LVFS). We evaluated diastolic trans-mitral flow in the 4 chambers view and measured the peak early diastolic trans mitral flow velocity $(\mathrm{E})$, the peak late diastolic trans mitral flow velocity (A) and ratio of maximum velocity of diastolic filling and maximum velocity of late diastolic filling through atrial contraction (E/A). With the help of tissue-Doppler (TDI) we could evaluate the peak mitral annulus systolic velocity (S'), peak early mitral annular diastolic velocity (E'), the peak late mitral annular diastolic velocity (A'), ratio of maximum velocity of diastolic filling and diastolic relaxation (E/E') and ratio of early mitral annular diastolic velocity and late mitral annular diastolic velocity (E'/A'). We completed the echocardiographic evaluation with the speckle-tracking method, which enabled us to determine global longitudinal strain (GLS) during LV contraction average GLS (GLS-AVG). By using this method in all three apical views we managed to obtain a "bull's-eye" map that represents LV segmental deformation. The echocardiographic examination was performed by the same examiner.

Vascular remodeling parameters. Arterial stiffness aortic pulse wave velocity (PWVAo) is a method of quantifying aortic rigidity. These patients had PWVAo analysis done using Medexpert Arteriograph ${ }^{\mathrm{TM}}$ TL2. This device enabled us to evaluate arterial dysfunction. The principle of the method consists of recording the signals of the pulse wave at the level of the brachial artery which is occluded for several seconds using an inflatable cuff. This investigation was done in a quiet room with the patient relaxed, without any stressful elements around. Using a measuring tape, the distance from the pubic bone to the sternal notch was determined. This, along with other information about the patient (age, height, etc.), was introduced into the computer. PWV is calculated using the distance traveled by the pulse wave in the aorta and the time of travel (RT/2). The measurements are wirelessly transmitted to the computer which then processes the information. To analyze the Intima-media thickness (IMT) the same General Electric Vivid E9 was used, the measurements being done with a $9 \mathrm{MHz}$ transducer (GE Healthcare). The IMT was determined at the level of the common carotid artery (CCA), $1 \mathrm{~cm}$ proximal to the carotid bulb. Ten measurements of the IMT of the posterior wall in the longitudinal plane were done, with a mean being calculated. The ankle-brachial index (ABI) was calculated with the help of a sphygmomanometer and a SonoTraX Vascular Doppler device (Edan Instruments, Langen, Germany). The brachial systolic blood pressure of both arms was determined, and, using the vascular Doppler device, the systolic pressure of the inferior limbs was recorded. This was equivalent to the pressure in the cuff at the moment of the first Doppler signal being detected in the dorsalis pedis artery. The ratio of systolic pressure of the upper limb to systolic pressure of the lower limb was calculated on both sides, with the greater value being recorded.

Statistical analysis. Statistical analysis was done using an SPSS program version 20.0 (IBM Corp, Armonk, NY, USA). The results are expressed in percentage and mean \pm standard deviation. The evaluation of systolic and diastolic left ventricular function, blood tests, heart rate, systolic and diastolic blood pressure and vascular parameters was done using the independent and paired $t$-tests. Values of $p<0.05$ were considered to have statistical significance.

\section{Results}

The evaluated patients were between 19 and 60 years old (45.38 \pm 11.27$), 15$ patients were female and had an average age of $55.67 \pm 18.32$ years, the other 20 patients $(57.1 \%)$ being male with an average age of $49.00 \pm 17.27$ years. All of these patients went through 2 cycles of chemotherapy between the first and second cardiovascular assessment. The cycles were administered in accordance with the national protocols/guidelines for the treatment of ALL and included prednisone, vincristine, and daunorubicin. More specifically, each chemotherapy cycle consisted of 3 days of daunorubicin 
at a dose of $398.74 \pm 53.61 \mathrm{mg} / \mathrm{m}^{2} /$ cycle, 14 days of prednisone at a dose of $1585.86 \pm 187.08 \mathrm{mg} / \mathrm{m}^{2} /$ cycle, and 4 days of vincristine at a dose of $8 \mathrm{mg} / \mathrm{cycle}$.

It was observed that all 35 patients suffered a significant weight loss during the 3 months of therapy, the BMI dropping from $25.57 \pm 3.91 \mathrm{~kg} / \mathrm{m}^{2}$ to $25.10 \pm 4 / 01 \mathrm{~kg} / \mathrm{m}^{2}$ $(p<0.05)$. There were no significant changes in SBP and DBP; however, heart rate was significantly higher after 3 months of therapy, increasing from $79.51 \pm 10.43 \mathrm{mmHg}$ to $84.68 \pm 14.13 \mathrm{mmHg}(p<0.05$; Table I).

Blood test analysis showed an increase in fasting blood glucose levels, from $102.82 \pm 20.30 \mathrm{mg} / \mathrm{dl}$ initially to $119.60 \pm 50.76 \mathrm{mg} / \mathrm{dl}$ at 3 months after chemotherapy treatment initiation. Another two parameters showed a statistically significant increase after 3 months: erythrocyte sedimentation rate (ESR) and D-dimers. ESR increased from $30.60 \pm 22.67 \mathrm{~mm} / \mathrm{h}$ to $33.74 \pm 23.47 \mathrm{~mm} / \mathrm{h}$, while D-dimers increased from $285.68 \pm 69.38 \mathrm{ng} / \mathrm{ml}$ to $377.20 \pm 176.92 \mathrm{ng} / \mathrm{ml}$ $(p<0.05)$. Other blood tests were also modified at 3 months, but without achieving statistical significance.

LV systolic function evaluation showed an important decrease in LVEF, from an initial value of $61.48 \pm 9.08 \%$ to a value of $54.05 \pm 7.77 \%$ after 3 months of chemotherapy $(p<0.001$; Figure 1). Mitral annular plane systolic excursion (MAPSE) at 3 months from the start of therapy decreased from $14.88 \pm 3.48 \mathrm{~mm}$ to $12.82 \pm 3.48 \mathrm{~mm}$. LVFS also suffered a significant change, from $41.63 \pm 12.30 \%$ to $35.40 \pm 12.09 \%$ $(p<0.001)$. The IVS had a slight growth between the time of the two measurements, LV end-diastolic diameter, and LV end-systolic diameter both had a statistically significant increases $(p<0.001)$, and so did LV end systolic volume $(p<0.001)$.

In regards to the LVEF of the 15 women that were part of the study group, there were significant changes at 3 months after treatment started. A decrease from $59.93 \pm 8.32 \%$ to $54.13 \pm 7.56 \%$ was observed $(p<0.001)$. The same trend existed for the 20 male patients enrolled, with a decrease of LVEF from $62.65 \pm 7.90 \%$ initially to $54.00 \pm 8.11 \%$ after the 2 cycles of chemotherapy $(p<0.001)$. Our data suggest that LVEF suffered was decreased following chemotherapy in both men and women.

Another observation is that of all the patients in the study, only 12, 6 of which were men and the other 6 women, had a decrease of LVEF to under $50 \%$ at the end of the 2 cycles of chemotherapy. This decrease was from an initial $55.16 \pm 3.51 \%$ to $45.66 \pm 3.79 \%$ after the 2 cycles $(p<0.001)$. These patients also suffered a significant loss of LVFS, from $41.66 \pm 13.54 \%$ at the start of therapy to $35.91 \pm 14.58 \%$ after 3 months $(p<0.05$; Table II).

Not only did the systolic function of the LV suffer changes during the 3 months of chemotherapy, the diastolic function was modified as well. Transmitral flow analysis demonstrated a larger $\mathrm{A}$ wave, a lower $\mathrm{E}$ wave, and a reduced $\mathrm{E} / \mathrm{A}$ ratio after
Table I. Evaluation of systolic and diastolic blood pressure, heart rate, $B M I$ at the start of treatment and 3 months after.

\begin{tabular}{lccc}
\hline Parameter & Baseline & After 3 months & $p$-Value \\
\hline BMI $\left(\mathrm{kg} / \mathrm{m}^{2}\right)$ & $25.57 \pm 3.91$ & $25.10 \pm 4.01$ & 0.006 \\
HR $(\mathrm{bpm})$ & $79.51 \pm 10.43$ & $84.68 \pm 14.13$ & 0.013 \\
SBP $(\mathrm{mmHg})$ & $126.97 \pm 10.75$ & $126.68 \pm 10.75$ & 0.918 \\
DBP $(\mathrm{mmHg})$ & $78.05 \pm 11.41$ & $76.60 \pm 11 / 47$ & 0.573 \\
\hline
\end{tabular}

BMI, Body mass index; HR, heart rate; SBP, systolic blood pressure; DBP, diastolic blood pressure. Data are the mean \pm standard deviation (SD).

Table II. Left ventricular systolic and diastolic function indicators for the 35 patients with acute lymphoblastic leukemia (ALL), measured at the start and after 3 months of chemotherapy.

\begin{tabular}{lccr}
\hline Parameter & Baseline & After 3 months & $p$-Value \\
\hline LVESV (ml) & $30.80 \pm 16.03$ & $37.80 \pm 16.74$ & $<0.001$ \\
LVEDV $(\mathrm{ml})$ & $77.05 \pm 26.54$ & $79.60 \pm 26.61$ & 0.248 \\
LVEDD (mm) & $47.60 \pm 6.50$ & $50.20 \pm 6.70$ & $<0.001$ \\
LVESD (mm) & $26.30 \pm 6.90$ & $29.30 \pm 8.10$ & $<0.001$ \\
LVEF (\%) & $61.48 \pm 8.08$ & $54.05 \pm 7.77$ & $<0.001$ \\
LVFS (\%) & $41.63 \pm 12.30$ & $35.40 \pm 12.09$ & $<0.001$ \\
IVS (mm) & $9.60 \pm 1.40$ & $10.30 \pm 1.30$ & $<0.001$ \\
LVPW (mm) & $9.60 \pm 1.30$ & $10.60 \pm 1.30$ & $<0.001$ \\
MAPSE (mm) & $14.88 \pm 3.55$ & $12.82 \pm 3.48$ & $<0.001$ \\
\hline
\end{tabular}

LVESV, Left ventricular end - systolic volume; LVEDV, left ventricular end - diastolic volume; EDD, end diastolic diameter; ESD, left ventricular end systolic diameter; EF, left ventricular ejection fraction; FS, fractional shortening; IVS, interventricular septum; PW, posterior wall; MAPSE, mitral annular plane systolic excursion. Data are the mean \pm standard deviation (SD).

Table III. The evaluation of transmitral diastolic flow and systolic function analysis through pulsed wave tissue-Doppler of the lateral mitral annulus, at the start of treatment and after 3 months of chemotherapy.

\begin{tabular}{lrrr}
\hline Parameter & \multicolumn{1}{c}{ Baseline } & After 3 months & $p$-Value \\
\hline E (m/s) & $0.86 \pm 0.23$ & $0.84 \pm 0.22$ & 0.464 \\
A (m/s) & $0.79 \pm 0.25$ & $0.81 \pm 0.26$ & 0.327 \\
E/A & $1.15 \pm 0.41$ & $1.10 \pm 0.41$ & 0.218 \\
$\mathrm{~S}^{\prime}(\mathrm{cm} / \mathrm{s})$ & $12.94 \pm 2.99$ & $11.66 \pm 3.09$ & 0.017 \\
$\mathrm{~A}^{\prime}(\mathrm{cm} / \mathrm{s})$ & $10.66 \pm 3.64$ & $9.43 \pm 2.85$ & 0.001 \\
$\mathrm{E}^{\prime}(\mathrm{cm} / \mathrm{s})$ & $11.51 \pm 4.21$ & $12.74 \pm 4.64$ & 0.010 \\
$\mathrm{E}^{\prime} / \mathrm{A}^{\prime}$ & $1.26 \pm 0.81$ & $1.51 \pm 0.93$ & 0.029 \\
$\mathrm{E}^{\prime} \mathrm{E}^{\prime}$ & $8.23 \pm 4.25$ & $7.45 \pm 3.68$ & 0.149 \\
\hline
\end{tabular}

E, Peak early diastolic trans mitral flow velocity; A, peak late diastolic trans mitral flow velocity; S', peak mitral annulus systolic velocity; E', peak early mitral annular diastolic velocity; A', peak late mitral annular diastolic velocity; E/A, ratio of maximum velocity of diastolic filling and maximum velocity of late diastolic filling through atrial contraction; E'/A', ratio of early mitral annular diastolic velocity and late mitral annular diastolic velocity; E/E', ratio of maximum velocity of diastolic filling and diastolic relaxation. Data are the mean standard deviation (SD). 

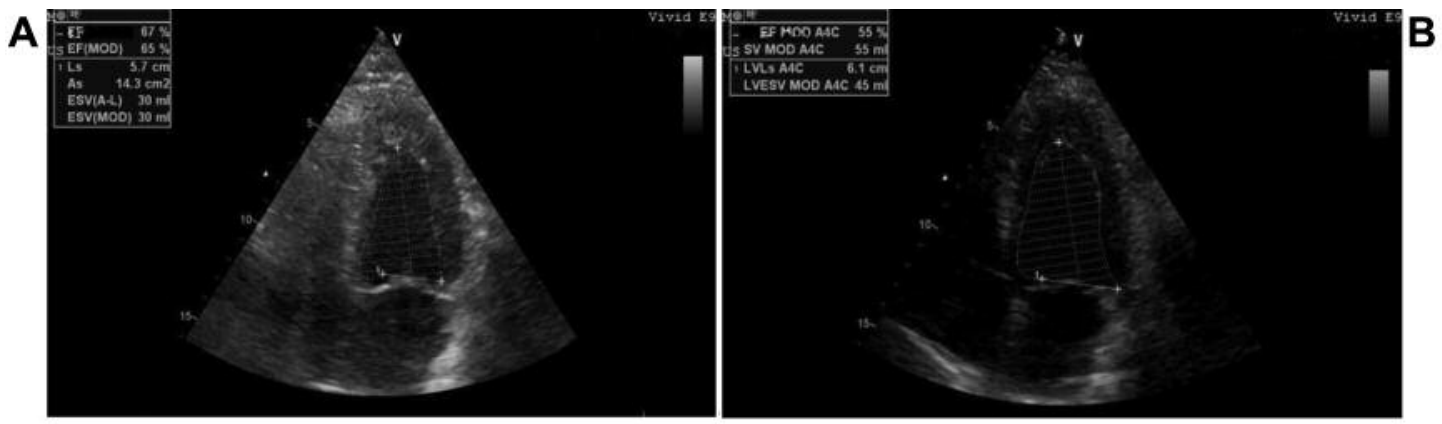

Figure 1. Assessment of left ventricular ejection fraction (LVEF) (\%) at baseline (A) and at 3 months after chemotherapy initialization (B) in a man with acute lymphoblastic leukemia enrolled in our study. LVEF decreased by $10 \%$ at 3 months after chemotherapy treatment.
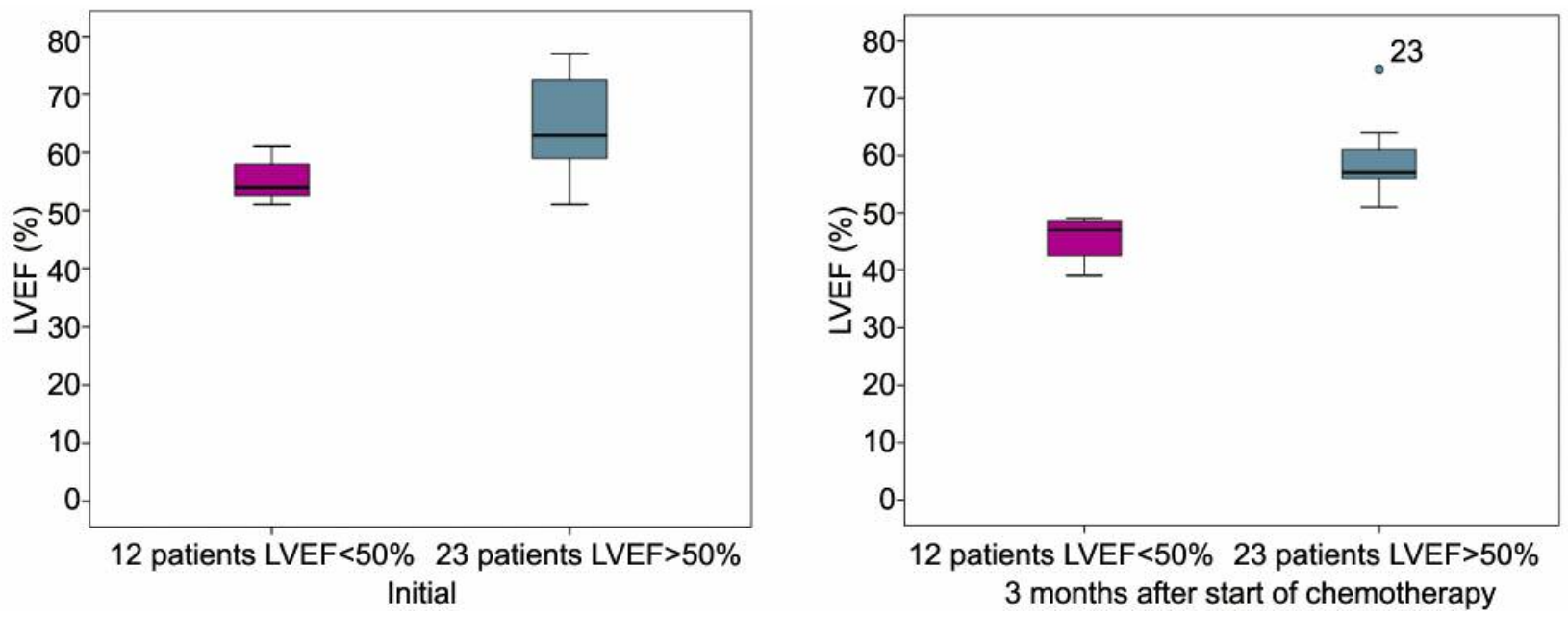

Figure 2. Left ventricular ejection fraction (LVEF) (\%) of patients with acute lymphoblastic leukemia (12 patients with LVEF $<50 \%$ after 2 chemotherapy cycles and 23 patients with LVEF $>50 \%$ after 2 chemotherapy cycles) at baseline and 3 months after chemotherapy initialization. The line represents the median value, the box represents the interquartile range, and the bars represent the minimum and maximum values. Points outside the boxplot represent outlier values.
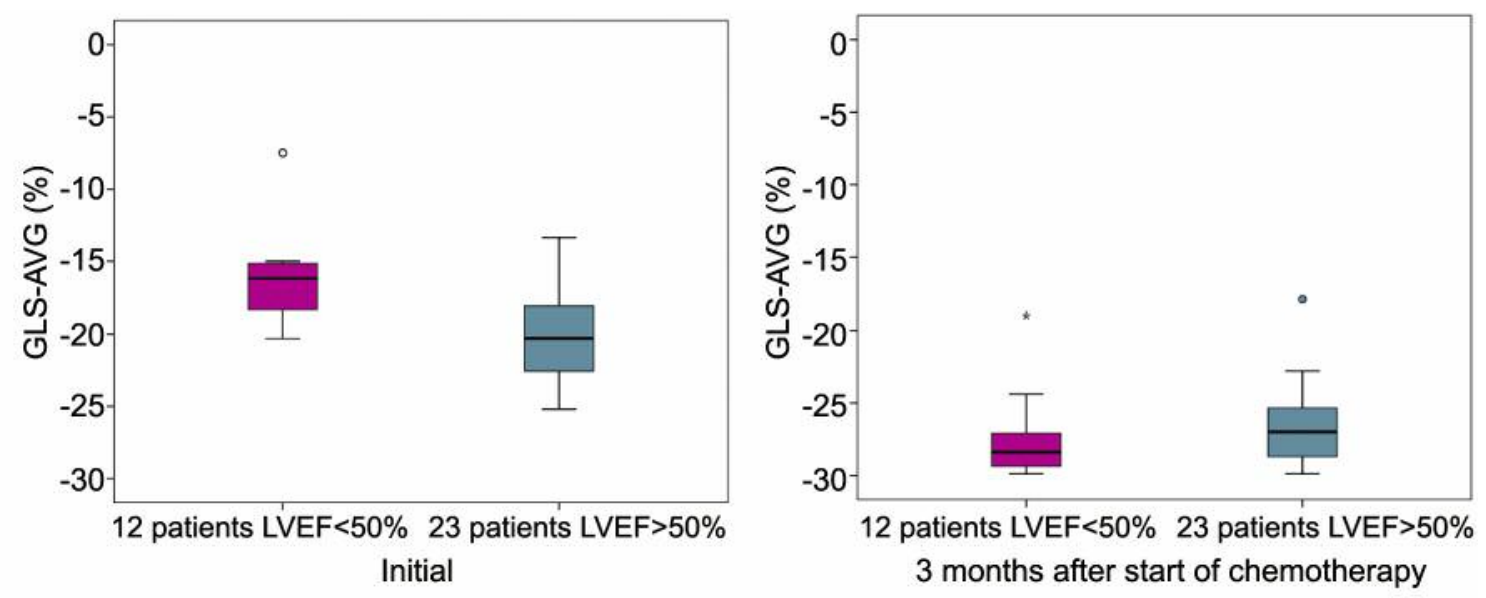

Figure 3. Speckle tracking: Average global longitudinal strain (GLS-AVG) (\%) of patients with acute lymphoblastic leukemia [12 patients with left ventricular ejection fraction $(L V E F)<50 \%$ after 2 chemotherapy cycles and 23 patients with LVEF>50\% after 2 chemotherapy cycles] at baseline and at 3 months after chemotherapy initialization. The line represents the median value, the box represents the interquartile range, and the bars represent the minimum and maximum values. Points outside the boxplot represent outlier values. 

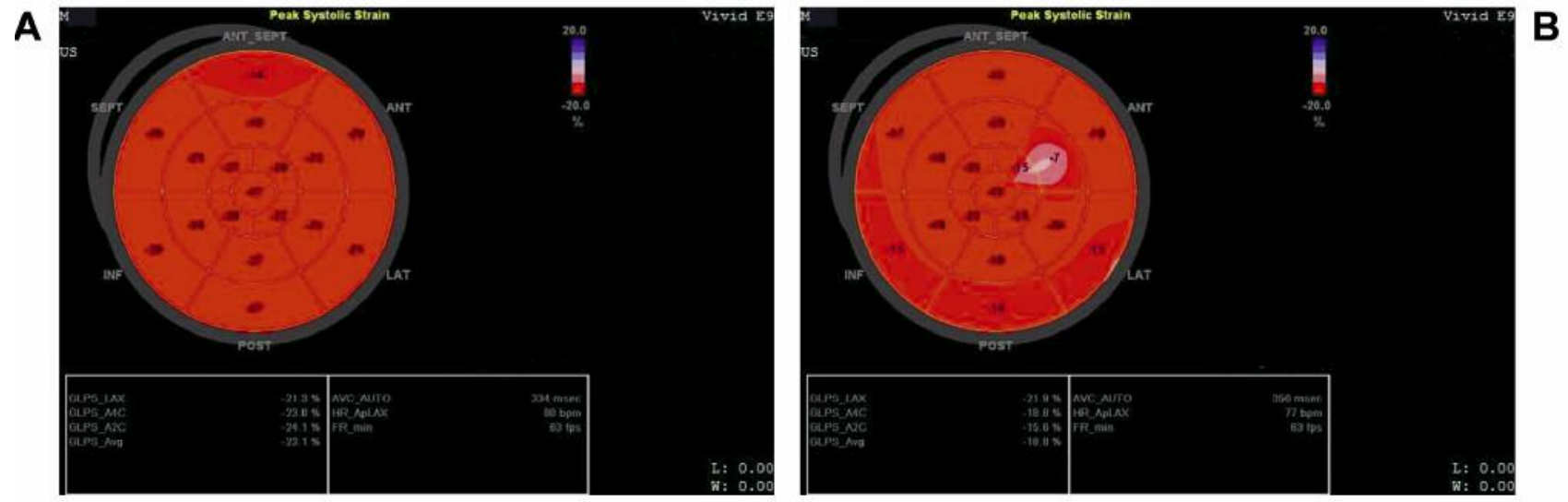

Figure 4. Speckle tracking: Global longitudinal strain $(G L S)$ initially and after 2 chemotherapy cycles in a patient with acute lymphoblastic leukemia. At the first determination, the values of the average GLS (GLS-AVG) (-23.1\%) were within the normal range (A). This shows that there were no changes in the structure and function of the left ventricle. After 3 months of chemotherapy, a decrease in the overall GLS-AVG (-18.8\%) was noted (B). This shows that there had been changes in myocardial structure from baseline. GLS-LAX, GLS of apical long axis; GLS-A4C, GLS of apical 4 chambers; GLS-A2C, GLS of apical 2 chambers.

2 cycles of therapy. Tissue-Doppler of the later mitral annulus revealed that maximum systolic velocity $S$ ' wave had a statistically significant decrease, from $12.94 \pm 2.99 \mathrm{~cm} / \mathrm{s}$ initially to $11.66 \pm 3.09 \mathrm{~cm} / \mathrm{s}(p<0.05)$. The E' wave increased significantly from $11.51 \pm 4.21 \mathrm{~cm} / \mathrm{s}$ to $12.74 \pm 4.64 \mathrm{~cm} / \mathrm{s}$ after 3 months of therapy, while the $A^{\prime}$ wave decreased with statistical significance from $10.66 \pm 3.64 \mathrm{~cm} / \mathrm{s}$ to $9.43 \pm 2.85$ $\mathrm{cm} / \mathrm{s}(p<0.05)$. The E'/A' ratio increased significantly over the 3 months of chemotherapy $(p<0.05)$. The E/E' also decreased in the time between the 2 evaluations (Table III)

Comparing the patients with a LVEF $<50 \%(n=12)$ with those with a LVEF $>50 \%(n=23)$ after 3 months of treatment, revealed that the first group had an initial LVEF significantly lower than the latter $(55.16 \pm 3.51 \%$ vs. $64.78 \pm 7.85 \%$, $p<0.001$; Figure 2). The patients with LVEF $<50 \%$ suffered a $10 \%$ loss of LVEF over the 3 months of chemotherapy $(45.66 \pm 3.79 \%$ after 2 cycles), compared with a $6 \%$ loss suffered by the 23 patients who had a LVEF $>50 \%$ $(58.43 \pm 5.26 \%$ after 2 cycles $)$.

By evaluating the parameters of vascular remodeling at 3 months, it was observed that the IMT was significantly increased compared to the first measurement that was taken before treatment $(p<0.001)$. IMT increased bilaterally in all patients after the second cycle chemotherapy. PWVAo was also significantly increased, from $6.93 \pm 1.02 \mathrm{~m} / \mathrm{s}$ initially to $7.93 \pm 1.05$ after treatment $(p<0.001)$. With the help of PWVAo we could also evaluate the patient vascular age, which saw an increase from $36.43 \pm 10.8$ years to $48.89 \pm 10.98$ years $(p<0.001)$. Although without statistical significance, the ABI decreased on the left side from $1.11 \pm 0.10$ to $10.08 \pm 0.13$, and on the right side from $1.16 \pm 0.28$ to $1.07 \pm 0.09$.
The separate analysis of the 12 patients that had a LVEF $<50 \%$ after chemotherapy revealed a PWVAo significantly higher than that of the other patients, reaching a value of $7.62 \pm 0.83 \mathrm{~m} / \mathrm{s}$ compared with the initial value of $6.65 \pm 0.70$ $\mathrm{m} / \mathrm{s}(p<0.001)$. IMT also increased with statistical significance, especially on the right side, where it grew to $0.86 \pm 0.24 \mathrm{~mm}$ from an initial value of $0.79 \pm 0.24 \mathrm{~mm}(p<0.05$; Table IV). With the use of the speckle tracking technique we evaluated LV global longitudinal strain (GLS). This was significantly reduced after the 2 cycle of chemotherapy, from an initial GLS of $-26.90 \pm 2.97 \%$ to $-18.37 \pm 4.12 \% \quad(p<0.001)$. These modifications were observed in both male and female patients. The 12 patients that had a LVEF $<50 \%$ after 3 months of chemotherapy had a statistically significant reduction in GLS, from $-27.45 \pm 3.10 \%$ to $-15.50 \pm 4.07 \%(p<0.001)$.

In regards to sex, GLS was significantly reduced in women, from an initial $-27.56 \pm 1.96 \%$ to $-18.23 \pm 3.50 \%$ after 3 months of treatment $(p<0.001)$, while men also suffered a reduction in GLS, from $-26.41 \pm 3.52 \%$ to $-18.47 \pm 4.62 \%$ after the 2 chemotherapy cycles $(p<0.001)$.

In patients with a 3 months LVEF $<50 \%$, LVEF and S' were both significantly reduced after chemotherapy. These results were significantly lower than those of patients with a post-chemotherapy LVEF $>50 \% \quad(p<0.05)$. Patients with LVEF $<50 \%$ after 2 cycles of chemotherapy had a statistically significant decrease in GLS at 3 months compared to the GLS at 3 months of patients with LVEF $>50 \%$ post-chemotherapy $(p<0.05)$.

Of the 12 patients with a LVEF $<50 \%$ at 3 months, 3 had received a dose of daunorubicin greater than 450 $\mathrm{mg} / \mathrm{m}^{2} /$ cycle. Of the 35 patients with ALL that were included 
Table IV. Assessment of hemodynamic and vascular parameters at baseline and 3 months after chemotherapy initialization.

\begin{tabular}{lccr}
\hline Parameter & Baseline & After 3 months & $p$-Value \\
\hline IMT left (mm) & $0.75 \pm 0.17$ & $0.82 \pm 0.17$ & $<\mathbf{0 . 0 0 1}$ \\
IMT right (mm) & $0.75 \pm 0.18$ & $0.83 \pm 0.14$ & $<\mathbf{0 . 0 0 1}$ \\
ABI left & $1.11 \pm 0.10$ & $1.08 \pm 0.13$ & 0.136 \\
ABI right & $1.16 \pm 0.28$ & $1.07 \pm 0.09$ & 0.067 \\
PWVAo (m/s) & $6.93 \pm 1.02$ & $7.93 \pm 1.05$ & $\mathbf{< 0 . 0 0 1}$ \\
Arterial age (years) & $36.43 \pm 10.80$ & $48.89 \pm 10.98$ & $<\mathbf{0 . 0 0 1}$ \\
\hline
\end{tabular}

IMT, Intima-media thickness of the carotid wall; ABI, ankle-brachial index; PWVAo, pulse wave velocity in the aorta. Data are the mean \pm standard deviation (SD). Statistically significant values are shown in bold.
Table V. Speckle tracking assessment trough global longitudinal strain (GLS) analyzed at baseline and 3 months after chemotherapy initialization.

\begin{tabular}{lccc}
\hline Parameter & Baseline & After 3 months & $p$-Value \\
\hline GLS-LAX (\%) & $-26.02 \pm 3.56$ & $-18.33 \pm 5.63$ & $<0.001$ \\
GLS-A4C (\%) & $-27.04 \pm 2.30$ & $-18.93 \pm 4.02$ & $<0.001$ \\
GLS-A2C (\%) & $-26.43 \pm 2.95$ & $-18.46 \pm 3.60$ & $<0.001$ \\
GLS-AVG (\%) & $-26.90 \pm 2.97$ & $-18.37 \pm 4.12$ & $<0.001$ \\
\hline
\end{tabular}

GLS-LAX, GLS of apical long axis; GLS-A4C, GLS of apical 4 chambers; GLS-A2C, GLS of apical 2 chambers; GLS-AVG, average GLS. Data are the mean \pm standard deviation (SD). in the study, 7 received a dose of daunorubicin greater than $450 \mathrm{mg} / \mathrm{m}^{2} /$ cycle. These patients suffered a significant drop in LVEF, from an initial $58.28 \pm 6.70 \%$ to $52.14 \pm 6.06 \%$ after 3 months of chemotherapy $(p<0.05)$. Speckle tracking examination of these patients revealed a statistically significant reduction in GLS from an initial value of $-27.87 \pm 1.78 \%$ to $-19.60 \pm 2.21 \%$ after 3 months of chemotherapy ( $p<0.001$; Figures 3 and 4 , Tables V and VI). An alteration of vascular remodeling parameters can also be observed in these patients. Right-side ABI experienced a statistically significant decrease from $1.18 \pm 0.09$ initially to $1.13 \pm 0.09$ at 3 months $(p<0.05)$. In addition, PWVAo significantly increased from an initial $6.38 \pm 0.26$ to $7.45 \pm 0.66$ after 3 months of chemotherapy $(p<0.05)$. This parameter can also be correlated with arterial age which increased from $30.86 \pm 9.29 \mathrm{~m} / \mathrm{s}$ to $42.71 \pm 8.59 \mathrm{~m} / \mathrm{s}$ after 3 months of chemotherapy $(p<0.001)$.

\section{Discussion}

Cardiotoxicity can be the most dangerous side effect of chemotherapy and depends mainly on the dose being used (3, 4). ALL is a haematologic disease that is caused by uncontrolled proliferation of immature cells called lymphoblasts in the bone marrow (8). Treatment is based on chemotherapeutic agents that can destroy the lymphoblasts. The type of treatment depends on the type of leukemia and the medical history of the patient (4). In this study left ventricular (LV) and vascular remodeling parameters were evaluated in 35 ALL patients, before treatment and 3 months after treatment initiation, during which period patients went through 2 cycles of chemotherapy. All patients received treatment schemes that included anthracyclines - daunorubicin. Anthracyclines are known for their cardiotoxic effects, especially in large doses, or when used in association with other chemotherapeutics or radiotherapy $(5,6)$. The dose of daunorubicin should not exceed $20 \mathrm{mg} / \mathrm{kg}$. It can be considered that for a cumulative dose of daunorubicin greater than $800 \mathrm{mg} / \mathrm{m}^{2}$ the incidence of heart failure exceeds $5 \%(3$, 9, 10).

Anthracyclines cause irreversible cardiac damage, and these effects do not always occur after the first doses of chemotherapy, sometimes effects may occur some years after treatment $(3,11-13)$.

There are several factors that may influence the occurrence of cardiotoxicity besides the chemotherapy dose: age, the presence of other comorbidities, cardiovascular risk factors $(3,14)$. If the signs of heart failure are discovered late, it is much more difficult to treat (15).

The patients we evaluated were administered 2 doses of daunorubicin between evaluations. No individual dose was greater than $500 \mathrm{mg} / \mathrm{m}^{2}$. 7 patients received a dose of daunorubicin greater than $450 \mathrm{mg} / \mathrm{m}^{2} /$ cycle. It was observed that of the 12 patients with LVEF $<50 \%$ after treatment, 3 received a dose of daunorubicin greater than $450 \mathrm{mg} / \mathrm{m}^{2} /$ cycle.

If patients receive increased doses of anthracyclines exceeding $240 \mathrm{mg} / \mathrm{m}^{2}$ and have an increased risk of cardiovascular disease, an assessment of cardiac function after this dose is required $(3,7,16,17)$.

Echocardiographic evaluation demonstrated that all patients had a reduction in LVEF after the 2 chemotherapy cycles with daunorubicin, an aspect observed in both men and women. Of all the study population, 12 patients had LVEF $<50 \%$ after therapy; their initial LVEF was lower than that of the rest patients, but still over $50 \%$. Many studies have shown that only $1 \%$ of patients who go through chemotherapy develop cardiotoxicity immediately after the first dose administration. This type of cardiotoxicity is most often reversible, but in most situations, it appears one year after treatment completion, or even later, after several years (18). Herein, all the patients that were studied developed subclinical cardiac dysfunction, none of them displaying clinical signs of heart failure. It is a possibility that this myocardial destruction progresses in time and cardiac 
Table VI. Evaluation of LVEF, S' and GLS baseline and at 3 months in patients with LVEF $<50 \%$ (after 2 chemotherapy cycles) compared with patients with $L V E F>50 \%$ (after 2 chemotherapy cycles).

\begin{tabular}{|c|c|c|c|}
\hline Parameter & $\begin{array}{c}\text { LVEF }<50 \% \text { (with cardiotoxicity), } \\
3 \text { months after start of treatment }(n=12)\end{array}$ & $\begin{array}{l}\text { LVEF }>50 \% \text { (without cardiotoxicity), } \\
3 \text { months after start of treatment }(n=23)\end{array}$ & $p$-Value \\
\hline LVEF (\%) initial & $55.16 \pm 3.51$ & $64.78 \pm 7.85$ & $<0.001$ \\
\hline LVEF (\%) 3 months after start of treatment & $45.66 \pm 3.79$ & $58.43 \pm 5.26$ & $<0.001$ \\
\hline$S^{\prime}(\mathrm{cm} / \mathrm{s})$ initial & $11.58 \pm 3.02$ & $13.65 \pm 2.77$ & 0.050 \\
\hline $\mathrm{S}^{\prime}(\mathrm{cm} / \mathrm{s}) 3$ months after start of treatment & $10.75 \pm 2.70$ & $12.13 \pm 3.23$ & 0.215 \\
\hline GLS $(\%)$ initial & $-27.45 \pm 3.10$ & $-26.62 \pm 2.93$ & 0.438 \\
\hline GLS (\%) 3 months after start of treatment & $-15.50 \pm 4.07$ & $-19.86 \pm 3.35$ & 0.002 \\
\hline
\end{tabular}

LVEF, Left ventricular ejection fraction; GLS, global longitudinal strain; S', peak mitral annulus systolic velocity. Data are the mean \pm standard deviation (SD).

manifestations appear much later, that is why periodic cardiac evaluation of these patients during and after remaining chemotherapeutic cycles is required. Current guidelines confirm the existence of this entity of heart failure, and recommend continuous evaluation during and after chemotherapy (18).

Tissue-Doppler confirmed the above results, demonstrating an S' wave that was significantly lower in all patients after 3 months of therapy. The results of Katarzyna and collaborators, who evaluated 35 patients receiving chemotherapy with anthracyclines using tissue-Doppler, revealed $S^{\prime}$ as the only parameter that indicated LV dysfunction (19). This aspect was mentioned in the existing cardio-oncology guidelines. The S' wave is an indicator of global systolic function, and its reduction indicates $\mathrm{LV}$ systolic dysfunction (20). The present results showed a reduction of $S^{\prime}$ in all the evaluated patients, supporting that the left ventricular systolic function that has suffered modifications, maybe earlier than it was expected; thus, explaining why using several methods of evaluating cardiotoxicity is recommended (21).

Several studies have shown that myocardial dysfunction appears after a reduction in GLS of more than $15 \%$ (17) and have underlined the fact that GLS can be an important predictor of cardiotoxicity (22). In the present study, we observed that GLS was decreased at the time of reevaluation in all patients; however, this reduction was not greater than $15 \%$. Even if the reduction did not reach the levels mentioned in the previous studies, this loss of GLS still indicates an irreversible deterioration of myocardial function that has yet to manifest itself clinically.

The role of echocardiography is extremely important, and the addition of other parameters, such as vascular remodeling, has been shown to increase the possibility of detecting the first signs of cardiotoxicity early on (23). The same study group revealed the importance of periodic evaluation of LVEF and GLS in patients who have received chemotherapy with anthracycline (22).
The evaluation of systolic and diastolic blood pressures did not reveal any significant changes, the values being within normal limits. Compared to the initial evaluation, heart rate was slightly increased, but none of the patients accused any symptoms possibly related to arrhythmias. It is known that anthracyclines can determine sinus tachycardia, so periodic cardiologic evaluations are needed (24).

PWVAo analysis proved an increase in all patients. Data from other studies suggests that PWVAo is a predictor of cardiovascular disease (3). PWVAo represents an increase in arterial rigidity. There are studies that have correlated the increase of PWVAo with strain modifications and LVEF decrease, after treatment with anthracyclines. Mihalcea et al. have demonstrated that anthracyclines can both alter cardiac function and increase arterial rigidity through the increase of cardiac after-load, aggravating mycordial dysfunction and reducing coronary artery perfusion (25). Moreover, it has been demonstrated that, following chemotherapy with anthracyclines, LVEF, longitudinal strain changes, and PWVAo had significant alterations (24).

Katarzyna and collaborators revealed that the 35 patients they followed, who were all receiving anthracycline treatment, had an increase in IMT. Monitoring this parameter could indicate the risk of cardiovascular events and, as some authors claim, could have a negative impact on left ventricular function (19). In the present study, all the patients had significantly increased IMT after the 2 chemotherapy cycles, suggesting early vascular damage.

In a study performed on a group of 85 patients with severe carotid artery stenosis, it was noted that patients who also had an LVEF $<50 \%$ in the echocardiographic evaluation were at a higher risk of developing a cardiovascular event, compared to those with LVEF $>50 \%$ (26). This may indicate that patients with cardiovascular disease and carotid atheromatosis may have increased risk of cardiovascular and cerebrovascular events after chemotherapy. It has also been shown that IMT and PWVAo are important indicators for assessing subclinical atherosclerosis $(3,21,27)$. 
In a previous study of 15 patients with leukemia and cardiovascular risk factors who received chemotherapy, there was a decrease in LVEF and an increase in IMT postchemotherapy treatment, especially in smokers and hypertensive patients. The authors suggested that correlation between cardiovascular risk factors and the calculation of IMT and LVEF is useful in determining the strategy and therapeutic adherence of patients with leukemia (28). We therefore consider it is important to evaluate these parameters periodically in patients receiving chemotherapy.

An analysis by Levato et al. revealed that $14.8 \%$ of a group of 82 chronic leukemia patients receiving chemotherapy developed peripheral arterial disease (29). Other authors have shown that the process of atherosclerosis may be more rapid in chronic myeloid leukemia patients who have received chemotherapy with a tyrosine kinase inhibitor. These results were demonstrated by Kim and his collaborators in a meta-analysis of 129 patients with leukemia. Among these patients, $6.3 \%$ developed peripheral arterial disease (30). These studies supported that following chemotherapy treatment there is a risk that patients will develop peripheral arterial disease more rapidly. In the present study, patients at 3 months had an ABI lower than the initial value, suggesting a process of accelerated atherosclerosis in patients undergoing chemotherapy.

Laboratory tests did not yield many modified parameters. ESR and D-dimers were elevated at the second determination. D-dimers could be used, as some authors suggest, identifying the risk of developing heart failure (31). Our results demonstrated that D-dimers should be evaluated periodically in patients receiving chemotherapy, in order to identify the first signs of heart failure. Another laboratory test parameter that showed changes was the fasting blood glucose level. This was increased after the 2 chemotherapy cycles, possibly due to the prednisone.

After viewing our results, we can conclude that modifications expressing LV dysfunction appear early on, even after the first doses of chemotherapeutics, and it is very important to identify these changes as quickly as possible. Moreover, the patients of the present study received 2 cycles of chemotherapy between evaluations, but their treatment continued afterwards; thus, further long-term evaluation is necessary to provide more information on cardiovascular disease and detection of cardiotoxicity in ALL.

\section{Conclusion}

Cardiovascular risk factors assessed in ALL patients before and after chemotherapy showed that cardiac dysfunction, once present, is most often irreversible. In addition to cardiac systolic function, vascular remodeling parameters were also severely affected, indicating that an accelerated atherosclerosis process may be induced by chemotherapy, even in young patients without any cardiovascular risk factors or previous cardiovascular pathology. Therefore, assessment of cardiovascular risk factors prior to treatment initiation should be considered as part of routine clinical evaluation in ALL patients. Early diagnosis of chemotherapy-induced cardiotoxicity may improve its treatment and lead to decreased mortality related to cardiovascular complications in patients with leukemia.

\section{Conflicts of Interest}

None declared.

\section{Authors' Contributions}

Anda Militaru, Marius Militaru, Stela Iurciuc, Sebastian Zus and Mircea Iurciuc clinically evaluated the patients and collected data used as comparative parameters of cardiovascular status before and after therapy. Daniel Lighezan validated clinical data. Anda Militaru wrote the paper. Anca Maria Cimpean and Petru Matusz revised the final draft of the manuscript, submitted the manuscript and managed all needed revisions.

\section{Acknowledgements}

Present work was supported by Romanian Society of Cardiology through its Atherothrombosis and Atherosclerosis Working Group.

\section{References}

1 Bloom MW, Hamo CE, Cardinale D, Ky B, Nohria A, Baer L, Skopicki H, Lenihan DJ, Gheorghiade M, Lyon AR and Butler J: Cancer therapy-related cardiac dysfunction and heart failure: Part 1: Definitions, pathophysiology, risk factors, and imaging. Circ Heart Fail 9(1): e002661, 2016. PMID: 26747861. DOI: 10.1161/CIRCHEARTFAILURE.115.002661.

2 Marinello J, Delcuratolo M and Capranico G: Anthracyclines as topoisomerase II poisons: From early studies to new perspectives. Int J Mol Sci 19(11): 3480, 2018. PMID: 30404148. DOI: 10.3390/ijms19113480

3 Zamorano JL, Lancellotti P, Muñoz DR, Aboyans V, Asteggiano R, Galderisi M, Habib G, Lenihan DJ, Lip GY, Lyon AR, Fernandez TL, Mohty D, Piepoli MF, Tamargo J, Torbicki A and Suter TM; ESC Scientific Document Group: 2016 ESC Position Paper on cancer treatments and cardiovascular toxicity developed under the auspices of the ESC Committee for Practice Guidelines: The Task Force for cancer treatments and cardiovascular toxicity of the European Society of Cardiology (ESC). Eur Heart J 37(36): 2768-2801, 2016. PMID: 27567406. DOI: 10.1093/eurheartj/ehw211

4 Early Breast Cancer Trialists' Collaborative Group (EBCTCG): Effects of chemotherapy and hormonal therapy for early breast cancer on recurrence and 15-year survival: an overview of the randomised trials. Lancet 365: 1687-1717, 2005. PMID: 15894097. DOI: 10.1016/S0140-6736(05)66544-0 
5 Reinbolt RE, Patel R, Pan X, Timmers CD, Pilarski R, Shapiro $\mathrm{CL}$ and Lustberg MB: Risk factors for anthracycline-associated cardiotoxicity. Support Care Cancer 24(5): 2173-2180, 2016. PMID: 26563179. DOI: 10.1007/s00520-015-3008-y

6 Rehammar JC, Jensen MB, McGale P, Lorenzen EL, Taylor C, Darby SC, Videbæk L, Wang Z and Ewertz M: Risk of heart disease in relation to radiotherapy and chemotherapy with anthracyclines among 19,464 breast cancer patients in Denmark, 1977-2005. Radiother Oncol 123(2): 299-305, 2017. PMID: 28365142. DOI: 10.1016/j.radonc.2017.03.012.

7 Hershman DL, McBride RB, Eisenberger A, Tsai WY, Grann VR and Jacobson JS: Doxorubicin, cardiac risk factors, and cardiac toxicity in elderly patients with diffuse B-cell non-Hodgkin's lymphoma. J Clin Oncol 26: 3159-3165, 2008. PMID: 18591554. DOI: $10.1200 / \mathrm{JCO} .2007 .14 .1242$

8 Ribera JM: Acute Lymphoblastic Leukemia. European LeukemiaNet Workpackage 6, 7:9, 2007. Available from: https:// www.leukemia-net.org/content/leukemias/all/project info/index_eng.html

9 Swain SM, Whaley FS and Ewer MS: Congestive heart failure in patients treated with doxorubicin: a retrospective analysis of three trials. Cancer 97: 2869-2879, 2003. PMID: 12767102. DOI: $10.1002 /$ cncr.11407

10 Bristow MR, Thompson PD, Martin RP, Mason JW, Billingham $\mathrm{ME}$ and Harrison DC: Early anthracycline cardiotoxicity. Am J Med 65: 823-832, 1978. PMID: 707541.

11 Felker GM, Thompson RE, Hare JM, Hruban RH, Clemetson DE, Howard DL, Baughman KL and Kasper EK: Underlying causes and long-term survival in patients with initially unexplained cardiomyopathy. N Engl J Med 342: 1077-1084, 2000. PMID: 10760308. DOI: 10.1056/NEJM200004133421502

12 Steinherz LJ, Steinherz PG, Tan CT, Heller G and Murphy ML: Cardiac toxicity 4 to 20 years after completing anthracycline therapy. JAMA 266: 1672-1677, 1991. PMID: 1886191.

13 Von Hoff DD, Layard MW, Basa P, Davis HL Jr., Von Hoff AL, Rozencweig M and Muggia FM: Risk factors for doxorubicininduced congestive heart failure. Ann Intern Med 91: 710-717, 1979. PMID: 496103.

14 Herrmann J, Lerman A, Sandhu NP, Villarraga HR, Mulvagh SL and Kohli M: Evaluation and management of patients with heart disease and cancer: cardio-oncology. Mayo Clini Proc 89(9): 1287-1306, 2014. PMID: 25192616. DOI: 10.1016/j.mayocp. 2014.05.013

15 Cardinale D, Colombo A, Lamantia G, Colombo N, Civelli M, De Giacomi G, Rubino M,Veglia F, Fiorentini C and Cipolla CM: Anthracycline-induced cardiomyopathy: clinical relevance and response to pharmacologic therapy. J Am Coll Cardiol 55: 213-220, 2010. PMID: 20117401. DOI: 10.1016/j.jacc.2009. 03.095

16 Drafts BC, Twomley KM, D’Agostino R Jr, Lawrence J, Avis N, Ellis LR, Thohan V, Jordan J, Melin SA,Torti FM, Little WC, Hamilton CA and Hundley WG: Low to moderate dose anthracycline-based chemotherapy is associated with early noninvasive imaging evidence of subclinical cardiovascular disease. JACC Cardiovasc Imaging 6: 877-885, 2013. PMID: 23643285. DOI: $10.1016 /$ j.jcmg.2012.11.017

17 Plana JC, Galderisi M, Barac A, Ewer MS, Ky B, ScherrerCrosbie M, Ganame J, Sebag IA, Agler DA, Badano LP, Banchs J, Cardinale D, Carver J, Cerqueira M, DeCara JM, Edvardsen T, Flamm SD, Force T, Griffin BP, Jerusalem G, Liu JE,
Magalhaes A, Marwick T, Sanchez LY, Sicari R, Villarraga HR and Lancellotti P: Expert consensus for multimodality imaging evaluation of adult patients during and after cancer therapy: a report from The American Society of Echocardiography and the European Association of Cardiovascular Imaging. Eur Heart J Cardiovasc Imaging 15: 1063-1093, 2014. PMID: 25239940. DOI: $10.1093 /$ ehjci/jeu 192

18 Çilsal E, Oğuz AD, Tunaoğlu FS, Kula S and Pektaş A: Subclinical reduction in left ventricular function using triplane and 2D speckle tracking echocardiography after anthracycline exposure in children. Anatol J Cardiol 19: 58-66, 2018. PMID: 29339701. DOI: 10.14744/AnatolJCardiol.2017.7944

19 Mizia-Stec K, Gościńska A, Mizia M, Haberka M, Chmiel A, Poborski W and Gąsior Z: Anthracycline chemotherapy impairs the structure and diastolic function of the left ventricle and induces negative arterial remodeling. Kardiologia Polska 71(7): 681-690, 2013. PMID: 23907900. DOI: 10.5603/KP.2013.0154

20 Kadappu KK and Thomas L: Tissue Doppler imaging in echocardiography: b value and limitations. Heart Lung Circ 24(3): 224-233, 2015. PMID: 25465516. DOI: 10.1016/ j.hlc.2014.10.003

21 Militaru A, Avram A, Cimpean AM, Iurciuc M, Matusz P, Lighezan D and Militaru M: The assessment of left ventricle function and subclinical atherosclerosis in patients with acute myeloid leukemia. In Vivo 32: 1599-1607, 2018. PMID: 30348722. DOI: 10.21873 /invivo. 11420

22 Jordan JH, Castellino SM, Melendez GC, Klepin HD, Ellis LR, Lamar Z, Vasu S, Kitzman DW, Nitm WO, Brubaker PH, Reichek N, D'Agostino RB Jr and Hundley WG: Left ventricular mass change after anthracycline chemotherapy. Circ Heart Fail 11(7): e004560, 2018. PMID: 29991488. DOI: 10.1161/ CIRCHEARTFAILURE.117.004560

23 Drafts BC, Twomley KM, D'Agostino R Jr, Lawrence J, Avis N, Ellis LR, Thohan V, Jordan J, Melin SA, Torti FM, Little WC, Hamilton CA and Hundley WG: Low to moderate dose anthracycline-based chemotherapy is associated with early noninvasive imaging evidence of subclinical cardiovascular disease. JAAC Cardiovascular Imaging 6(8): 877-885, 2013. PMID: 23643285. DOI: 10.1016/j.jcmg.2012.11.017

24 Boriani G, Fauchier L, Aguinaga L, Beattie JM, Blomstrom Lundqvist C, Cohen A, Dan GA, Genovesi S, Israel C, Joung B, Kalarus Z, Lampert R, Malavasi VL, Mansourati J, Mont L, Potpara T, Thornton A and Lip GYH ; ESC Scientific Document Group: European Heart Rhythm Association (EHRA) consensus document on management of arrhythmias and cardiac electronic devices in the critically ill and post-surgery patient, endorsed by Heart Rhythm Society (HRS), Asia Pacific Heart Rhythm Society (APHRS), Cardiac Arrhythmia Society of Southern Africa (CASSA), and Latin American Heart Rhythm Society (LAHRS). Europace 21(1): 7-8, 2019. PMID 29905786. DOI: 10.1093/europace/euy110

25 Mihalcea DJ, Florescu M, Suran BM, Enescu OA, Mincu RI, Magda S, Patrascu N and Vinereanu D: Comparison of pulse wave velocity assessed by three different technique: Arteriograph, Complior, and Echo-tracking. Heart Vessels 31(4): 568-577, 2016. PMID: 25633054. DOI: 10.1007/s00380-015-0632-x

26 Militaru M, Militaru A, Lighezan D, Petrescu L and Simu M: Prognostic criteria and treatment for patients candidates for surgery or percutaneous carotid reperfusion. Rom J Neurol 13(2): 60-68, 2014. 
27 Hussain S M, Oldenburg B, Wang Y, Zoungas S and Tonkin AM: Assessment of cardiovascular disease risk in South Asian populations. Int J Vasc Med 2013: 786801, 2013. DOI: $10.1155 / 2013 / 786801$.

28 Militaru M, Militaru AG, Lighezan D and Simu M: Correlation between vascular risk factors, arterial remodeling and systolic function in patients with leukemia. Eur J Neurol 25(Suppl. 2): 547,2018

29 Levato L, Cantaffa R, Kropp MG, Magro D, Piro E and Molica S: Progressive peripheral arterial occlusive disease and other vascular events during nilotinib therapy in chronic myeloid leukemia: a single institution study. Eur J Haematol 90: 531-532, 2013. PMID: 23506097. DOI: 10.1111/ejh.12096
30 Kim TD, Rea D, Schwarz M, Grille P, Nicolini FE, Rosti G, Levato L, Giles FJ, Dombret H, Mirault T, Labussieri H, Lindhorst R, Havercamp W, Buschmann I and Dorken: Peripheral artery occlusive disease in chronic phase chronic myeloid leukemia patients treated with nilotinib or Imatinib. Leukemia 27(6): 13161321, 2013. PMID: 23459449. DOI: 10.1038/leu.2013.70

31 Danesh J, Whincup P, Walker M, Lennon L, Thomson A, Appleby P, Rumley A and Lowe GD: Fibrin D-dimer and coronary heart disease. Circulation 103(19): 2323-2327, 2001. PMID: 11352877.

Received April 8, 2019

Revised May 21, 2019

Accepted May 23, 2019 